\title{
Mechanical Properties and Piezoresistivity of Tellurium Nanowires
}

Sijia Ran ${ }^{l}$, Tom S. Glen ${ }^{l,}{ }^{\dagger}$, Bei Li ${ }^{1}$, Tianye Zheng ${ }^{1}$, In-Suk Choi ${ }^{2}$, Steven T. Boles ${ }^{1, *}$

${ }^{1}$ Department of Electrical Engineering, The Hong Kong Polytechnic University, Kowloon, Hong Kong SAR, China

${ }^{2}$ Department of Materials Science and Engineering, Seoul National University, Seoul 08826, Republic of Korea

* Corresponding author e-mail: steven.t.boles@ polyu.edu.hk 


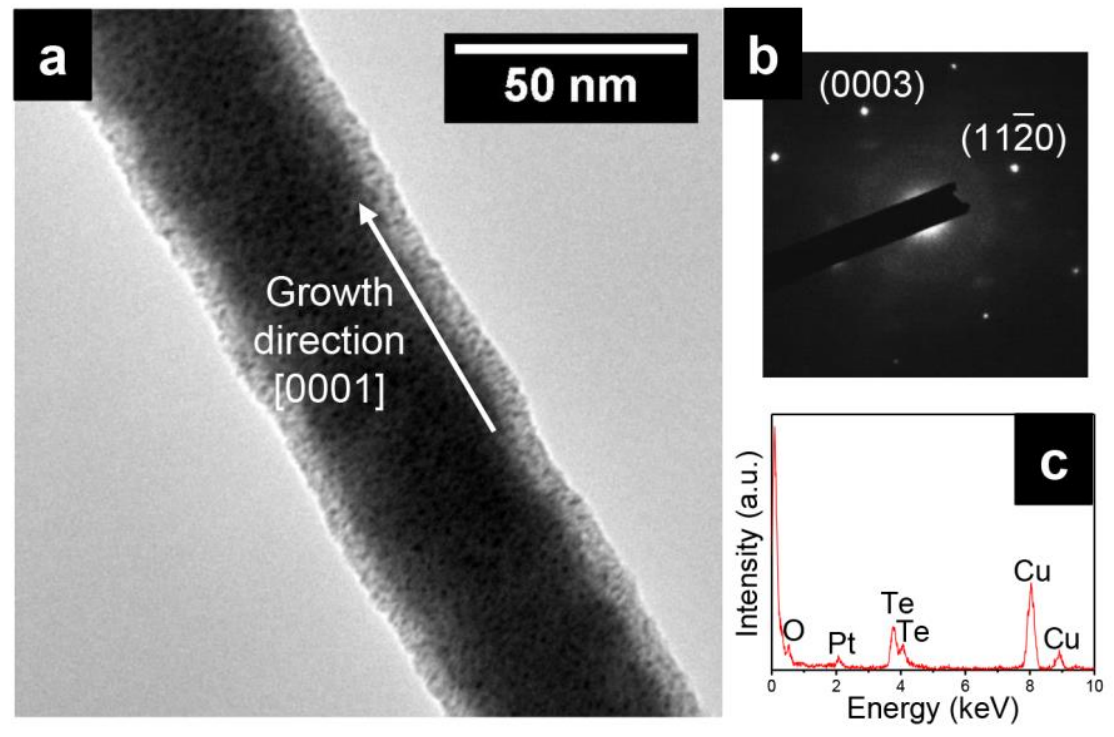

Figure S1. (a) BF TEM image of a harvested NW with a surface coating. (b) and (c) show the corresponding SAED pattern and the EDX spectrum of the wire shown in (a). Diffraction pattern indicates the NW is a single crystal with hexagonal structure and [0001] growth direction. EDX spectrum shows the composite nature $(\mathrm{Te}, \mathrm{C} / \mathrm{Pt})$ of the harvested $\mathrm{NW}$. The $\mathrm{C} / \mathrm{Pt}$ surface coating is formed during TEM sample preparation. 